\title{
Recent advances in minimally invasive surgery for gynecologic indications
}

\author{
Yu-Jin Koo \\ Department of Obstetrics and Gynecology, Yeungnam University College of Medicine, Daegu, Korea
}

Recently, an increasing interest in less invasive surgery has led to the advent of laparoendoscopic singlesite surgery (LESS) and natural orifice transluminal endoscopic surgery (NOTES). LESS and NOTES could be technically challenging, but available literature has demonstrated the feasibility and safety of LESS for benign gynecologic diseases. However, the evidence is not strong enough to recommend the use of LESS over that of conventional multiport laparoscopic surgery (MLS). As per the results of the most recently published meta-analysis, the majority of surgical outcomes are equivalent between LESS and MLS, except for the longer operative time in LESS for both adnexal surgery and hysterectomy. Although an increasing number of studies have reported on robotic LESS, NOTES, and LESS for gynecologic malignancy, definite conclusions have not been drawn owing to the lack of sufficient information.

Keywords: Laparoscopy; Minimally invasive surgical procedures; Natural orifice endoscopic surgery

\section{INTRODUCTION}

Over the last two decades, minimally invasive surgery has been widely adopted, and it has become the standard procedure in gynecologic diseases. Considerable evidence indicates that owing to the non-requirement of a large abdominal incision, laparoscopic surgery offers substantial advantages over open surgery, including less postoperative pain, shorter hospital stays, faster postoperative recovery, improved cosmetic outcomes, fewer wound-related complications, and lower costs [1,2].

An increasing interest in even less invasive surgery has led to the advent of laparoendoscopic single-site surgery (LESS), which is also called single-port surgery or single-incision lapa-

Received: September 5, 2018, Revised: September 28, 2018 Accepted: October 1, 2018

Corresponding Author: Yu-Jin Koo, Department of Obstetrics and Gynecology, Yeungnam University College of Medicine, 170, Hyeonchung-ro, Nam-gu, Daegu 42415, Korea

Tel: +82-53-620-3433, Fax: +82-53-654-0676

E-mail: yujinkoo@yu.ac.kr roscopy. During this procedure, all instruments are inserted through a single skin incision, which is almost invariably made at the umbilicus. Historically, gynecology has played a leading role in the development of LESS. The first reported case of LESS was a tubal sterilization performed by Wheeless in 1969 [3]. Approximately 20 years later, Pelosi and Pelosi [4] reported the first case of hysterectomy through LESS. Currently, LESS is used for various surgeries for gynecologic, urologic, and gastrointestinal indications.

A single small incision of entry could pose technical problems to the surgeon. However, a growing body of knowledge indicates that the feasibility and safety of LESS are currently evident for more complex procedures than they were in the past, although the clinical advantages of LESS over conventional multiport laparoscopic surgery (MLS) are still under investigation.

This study aimed to provide a systematic review of the recent updates on the clinical outcomes of minimally invasive procedures in gynecologic diseases. We particularly focused on the procedure of not only LESS but also natural orifice transluminal endoscopic surgery (NOTES), which is an emerging technique in minimally invasive surgery.

Copyright (C) 2018 Yeungnam University College of Medicine

This is an Open Access article distributed under the terms of the Creative Commons Attribution Non-Commercial License (http://creativecommons.org/licenses/by-nc/4.0/) which permits unrestricted non-commercial use, distribution, and reproduction in any medium, provided the original work is properly cited. 


\section{LESS in benign adnexal diseases}

Soon after the introduction of LESS, several studies have proposed the promising benefits of LESS over MLS, such as improved cosmesis, less pain, and decreased incisional morbidity [5-7]. The hypothesis was similar to that of the benefits of MLS over open surgery. However, the superiority of LESS over MLS is still debatable.

In fact, recent data do not support the added advantages of LESS over MLS in both adnexal surgery and hysterectomy, which are the common gynecologic procedures [8-11]. In 2017, Schmitt et al. reported a meta-analysis evaluating the clinical advantages of LESS for adnexal surgery [8]. Through a pooled analysis of six randomized controlled trials (RCTs), the authors showed that there was no significant difference between LESS and MLS in terms of length of hospital stay, blood loss, postoperative pain, and cosmetic outcomes. In fact, the operative time was longer for LESS ( $p=$ 0.03 ). No cases of conversion to open surgery were identified in either of the groups. Consequently, the authors concluded that LESS cannot be recommended in current practice for adnexal surgery in the absence of significant differences between LESS and MLS.

Prior to the study by Schmitt et al., one meta-analysis [10] had compared LESS and MLS, although it included hysterectomy as well as adnexal surgery for benign indications. The authors found no significant difference in the risk of complications between LESS and MLS. Notably, the mean operative time was 6.9 minutes longer for adnexal surgery performed by LESS than that for surgery performed by MLS. The authors concluded that the evidence is not strong enough to recommend the use of LESS over MLS, even though the difference may not be clinically significant, and the quality of this evidence was poor.

To date, studies have reported inconsistent results with regard to the cosmetic outcomes of LESS and MLS $[8,12,13]$. Unexpectedly, a recent meta-analysis by Schmitt et al. found no significant difference in cosmetic outcomes between patients who underwent LESS and those who underwent MLS $(p=0.71)$ [8]. In another study on MLS for benign gynecologic surgery, patients were postoperatively interviewed regarding their skin wound [14]. The majority of the patients remembered fewer incisions than those actually made, and pre- ferred to eliminate, if possible, the umbilical incision among the four skin incisions. This result indicates a limited cosmetic advantage of LESS.

The main drawbacks of LESS are the requirement of a larger umbilical incision (up to $2.5-3 \mathrm{~cm}$ ) and the technical challenges associated with the procedure, especially owing to the lack of triangulation caused by the proximity of the instruments. There is also a concern regarding port herniation owing to the larger opening in LESS, although the risk has not fully been determined owing to its rare occurrence [15-17]. Paradoxically, it is expected that a single incision using an open technique in LESS may decrease the risk of incisional morbidity [11]. Moreover, the larger umbilical incision is useful for specimen extraction and extracorporeal procedures such as omentectomy and repair of small bowel.

The technical challenge associated with LESS, especially for advanced surgery, is a well-recognized disadvantage. Although an indication of LESS is very uncertain and is largely dependent on the skill and preference of the operating surgeon, it is suggested that LESS should be avoided as an initial experience of surgeons for more complex surgeries, such as large ovarian cystectomy, surgery for severe endometriosis $[11,18]$, and tubo-ovarian abscess combined with severe pelvic adhesion, and in women with a history of panperitonitis and morbidly obese women.

Few studies have evaluated the cost-effectiveness of LESS. The cost analysis is complicated and is influenced by various factors, such as the insurance program of each country. Although LESS surgery does not require two or three ancillary trocars, the possible use of a multi-channel commercial trocar incurs additional costs [19].

\section{LESS in benign uterine diseases}

Hysterectomy may require a higher level of surgical skill than adnexal surgery does. Nevertheless, LESS is now widely applied for not only laparoscopic-assisted vaginal hysterectomy (LAVH) but also total laparoscopic hysterectomy (TLH). In 2015 , a retrospective study reported that $80 \%$ of hysterectomies in a single hospital in Korea were performed via LESS [20].

In 2017, Sandberg et al. published a meta-analysis that included RCTs, and prospective and retrospective cohort stu- 
dies for evaluating LESS versus MLS for hysterectomy in benign disease [21]. No significant differences were observed in the complication rates, postoperative pain, intraoperative blood loss, and length of hospital stay between the two groups. The complication rate was 5.3\% in the LESS group and 5.6\% in the MLS group for major complications, and 3.4\% in the LESS group and $4.5 \%$ in the MLS group for minor complications. No case of port herniation was found. Based on subanalysis specific for LAVH and TLH, no difference in complication rate was observed between the groups. The rate of conversion to open surgery was $1.2 \%$ in the LESS group and $0.4 \%$ in the MLS group, with no significant difference; however, $3.5 \%$ of patients who underwent LESS required an additional port. The operative time was 11.3 minutes longer in the LESS group than in the MLS group, which is in line with the results of the meta-analysis by Schmitt et al., which evaluated adnexal surgery [8].

In terms of learning curve, previous studies indicated that even well-trained laparoscopic surgeons must perform at least 10 to 15 [6] and up to 40 surgeries [22] using LESS-TLH, in order to acquire sufficient skills. In particular, vaginal vault suture, uterine myomectomy, and LESS hysterectomy for increased uterine weight are considered challenging. In 2017, a review article suggested that minimally invasive hysterectomy for the large uterus is feasible [23]. However, only two RCTs comparing LESS and MLS were included in the analysis; moreover, women with uterine size greater than 18 weeks were excluded.

Owing to inadequate triangulation and instrumental collision, closure of the vaginal cuff is particularly difficult during LESS-TLH. The lack of consensus regarding the best technique for cuff closure is evidenced by the various approaches used by individual surgeons; however, new techniques and materials have currently improved surgical proficiency and the strength of closure. For example, barbed suture, which is a type of knotless suture, has gained popularity as a useful technique during LESS. Several studies have reported a shorter operative time and decreased risks of postoperative vaginal bleeding and vaginal cuff cellulitis in cases where a barbed suture is used, compared with cases where a conventional suture is used, although data from other studies are inconsistent [24]. However, issue about the potential safety of barbed sutures was recently raised by the Government of Canada, which warned about the risk of small-bowel obstruction, pro- bably caused by hooking onto a part of the small intestine by a portion of the barbed suture.

Myomectomy via LESS is one of the most demanding procedures. The entire process, including myoma extraction, bleeding control during myoma enucleation, closure of uterine incision, and morcellation of myoma, is challenging. However, in 2017, a comparative study consisting of 100 cases of LESS versus MLS showed the feasibility and safety of LESSmyomectomy with respect to operative time, postoperative pain, blood loss, and length of hospital stay [25]. Further large multicentric studies are required to ascertain the surgical advantages of LESS over MLS in myomectomy.

\section{LESS in gynecologic malignancies}

In an effort to achieve the benefits of minimally invasive techniques, many clinicians have implemented LESS, even for oncologic surgeries. The first report of LESS for gynecologic oncology procedures was published by Fader and Escobar in 2009 [26, in which the surgical indications included various gynecologic cancers and precancerous conditions. Thereafter, several studies have demonstrated the feasibility and safety of LESS in gynecologic oncology.

In a retrospective multicentric study, Fagotti et al. evaluated the effect of LESS in 100 cases of early-stage endometrial cancer [27]. This was a large-scale study of endometrial cancer treated using LESS. Pelvic and paraaortic lymphadenectomy was performed in 48 and 27 patients, respectively, and a median of 16 pelvic nodes and 7 paraaortic lymph nodes were retrieved. Eight cases of intra- or postoperative complications were found, and two conversions to MLS or open surgery were observed. Altogether, the authors concluded that LESS is feasible for treatment of early-stage endometrial cancer.

Similarly, the first case of LESS radical hysterectomy for cervical cancer was reported by Garrett and Boruta in 2012 [28], and the same study group reported the first retrospective series of patients with stage I cervical cancer who were treated using LESS radical hysterectomy and pelvic lymphadenectomy [29]. The surgical outcomes were promising, as follows: 260 minutes of median operative time, $60 \mathrm{~mL}$ of median blood loss, and $9 \%$ of conversion rate to MLS or open surgery. Finally, the authors proposed larger studies to determine whether LESS can be used in routine gynecologic 
oncology practice.

Meanwhile, a wide peritoneal spread and frequent recurrence of ovarian cancer have limited the use of LESS. Unlike endometrial or cervical cancer, the feasibility and oncologic safety of even MLS for early-stage ovarian cancer is still under debate. It might be plausible that LESS is used merely for initial diagnosis and intra-abdominal exploration, but not for upfront surgical staging, in ovarian cancer. To date, no series of ovarian cancer treated with LESS has been reported.

\section{Robotic-assisted LESS (R-LESS) in gynecologic surgery}

Although the surgical indication for MLS has slowly extended to more advanced gynecologic pathologies, robotic minimally invasive surgery has been rapidly utilized in various gynecologic diseases. Less than 10 years ago, the first report of R-LESS in gynecology introduced risk-reducing bilateral salpingo-oophorectomy and total hysterectomy performed in a woman with breast cancer [30]. In 2014, the Food and Drug Administration approved R-LESS instruments for use in benign hysterectomy and salpingo-oophorectomy. Recently, RLESS is becoming more standardized and is increasingly used by surgeons despite a technical limitation attributed to the absence of EndoWrist technology, unlike the robotic MLS platform [31].

In 2018, a systemic review described the surgical outcomes of 810 cases of R-LESS hysterectomy performed for non-neoplastic disease [31]. Although the operative time of and blood loss associated with the procedure were tolerable, definite conclusions regarding postoperative pain and cosmetic results remained unknown owing to the lack of sufficient information. Complications and conversion to open surgery were reported in $4.9 \%$ and $2.8 \%$ of the patients, respectively. In terms of learning curve, the study found that a proficiency in vaginal cuff suture can be achieved after 14 cases, and that both large uterus and previous abdominal surgery are limitations of R-LESS hysterectomy.

A previous study by Lopez et al. retrospectively compared R-LESS and LESS hysterectomy for benign indications in 100 patients [32]. The rate of conversion to multiport procedures was $16 \%$ in the R-LESS group and 10\% in the LESS group, without a statistically significant difference. There were no conversions to open surgery. Blood loss was equivalent between the two groups; however, the operative time was 24.9 minutes longer in the R-LESS group.

Several studies have shown the feasibility and safety of RLESS procedures. However, most of these studies are case series or retrospective cohort studies. Further evidence in this regard is warranted.

\section{NOTES in gynecologic surgery}

NOTES is a scarless single-entry surgery, which is an emerging concept in minimally invasive techniques. Although it can be performed via various access routes such as the stomach, esophagus, vagina, bladder, and rectum, the vagina has been focused on almost exclusively, as it might be the safest and most feasible route for clinical application [33]. Reported advantages of NOTES include the absence of a visible abdominal scar, less operative pain, shorter hospital stay, improved operative visibility, and possibly, no requirement of adhesiolysis to expose the pelvic organs [33].

In 2013, Yang et al. reported seven cases of transvaginal NOTES for salpingo-oophorectomy [34]. The median tumor size was $6 \mathrm{~cm}$; the estimated blood loss was minimal, and the mean operative time was 45 minutes. No postoperative complication or conversion to standard laparoscopic surgery was reported, suggesting that transvaginal NOTES is feasible and safe for the treatment of adnexal masses.

More recently, a meta-analysis evaluated the advantages and disadvantages of NOTES hysterectomy in patients with benign gynecological disease [35]. The study did not find RCTs but included two retrospective cohort studies comparing NOTES hysterectomy with conventional LAVH (either LESSor MLS-LAVH). It was observed that compared to conventional LAVH, the NOTES group was associated with shorter operative time and hospital stay but higher cost. There were no differences between the groups in terms of intra- or postoperative complications and postoperative pain. No case of conversion to conventional laparoscopy or open surgery was demonstrated. The study concluded that NOTES should be considered as an option for gynecological approaches.

Although the use of NOTES in gynecologic surgery is rapidly increasing, technical difficulties that must be overcome still exist. As the limitation of NOTES is mainly related to 
collision between instruments, a study recommended using a flexible scope or a rigid scope with at least a 30-degree lens [33].

\section{CONCLUSION}

LESS and NOTES are the emerging techniques in the evolution of minimally invasive surgery. Their technical limitations continue to lessen with the recent technological innovations, such as the robotic surgical system, various types of multi-channel ports, and articulating instruments.

The feasibility and safety of LESS for benign gynecologic diseases have been demonstrated. However, the evidence is not strong enough to recommend the use of LESS over MLS, as the definitive superiority of LESS has not been established. Based on the result of the most recently published meta-analysis, the majority of surgical outcomes are equivalent between LESS and MLS, except for the longer operative time required for LESS than for MLS for both adnexal surgery and hysterectomy. Although several studies have been reporting on robotic LESS, NOTES, and LESS for gynecologic malignancy, definite conclusions with regard to their safety and efficacy have not been drawn owing to the lack of information.

The clinically relevant benefits and risks of LESS could vary according to the individual institution or surgeon. The available literature highlights the statistical limitation of the analysis as well as the major differences in the surgical techniques and the equipment used among the studies published.

Extensive experience with minimally invasive surgery, especially MLS, has already been accumulated. However, future well-designed large-scale research is necessary to determine the effectiveness of LESS as well as R-LESS and NOTES for gynecologic malignancies. An indication and absolute contraindication should also be ascertained before the widespread use of these procedures.

\section{CONFLICT OF INTEREST}

No potential conflict of interest relevant to this article were reported.

\section{ORCID}

Yu-Jin Koo, https://orcid.org/0000-0002-5114-8000

\section{REFERENCES}

1. Nieboer TE, Johnson N, Lethaby A, Tavender E, Curr E, Garry R, et al. Surgical approach to hysterectomy for benign gynaecological disease. Cochrane Database Syst Rev 2009;(3): CD003677.

2. Aarts JW, Nieboer TE, Johnson N, Tavender E, Garry R, $\mathrm{Mol} \mathrm{BW}$, et al. Surgical approach to hysterectomy for benign gynaecological disease. Cochrane Database Syst Rev 2015;(8): CD003677.

3. Wheeless CR. A rapid, inexpensive, and effective method of surgical sterilization by laparoscopy. J Reprod Med 1969;3: 65-9

4. Pelosi MA, Pelosi MA 3rd. Laparoscopic hysterectomy with bilateral salpingo-oophorectomy using a single umbilical puncture. N J Med 1991;88:721-6.

5. Fader AN, Cohen S, Escobar PF, Gunderson C. Laparoendoscopic single-site surgery in gynecology. Curr Opin Obstet Gynecol 2010;22:331-8.

6. Escobar PF, Starks D, Fader AN, Catenacci M, Falcone T. Laparoendoscopic single-site and natural orifice surgery in gynecology. Fertil Steril 2010;94:2497-502.

7. Mencaglia L, Mereu L, Carri G, Arena I, Khalifa H, Tateo S, et al. Single port entry - are there any advantages? Best Pract Res Clin Obstet Gynaecol 2013;27:441-55

8. Schmitt A, Crochet P, Knight S, Tourette C, Loundou A, Agostini A. Single-port laparoscopy vs conventional laparoscopy in benign adnexal diseases: a systematic review and meta-analysis. J Minim Invasive Gynecol 2017;24:1083-95.

9. Carbonnel M, Revaux A, Frydman R, Yazigi A, Ayoubi JM. Single-port approach to benign gynecologic pathology. A review. Minerva Ginecol 2015;67:239-47.

10. Murji A, Patel VI, Leyland N, Choi M. Single-incision laparoscopy in gynecologic surgery: a systematic review and metaanalysis. Obstet Gynecol 2013;121:819-28.

11. Boruta DM. Laparoendoscopic single-site surgery in gynecologic oncology: An update. Gynecol Oncol 2016;141:616-23.

12. Song T, Park JY, Kim TJ, Lee YY, Choi CH, Lee JW, et al. A prospective comparative study of cosmetic satisfaction for three different surgical approaches. Eur J Obstet Gynecol Reprod Biol 2015;190:48-51.

13. Eom JM, Ko JH, Choi JS, Hong JH, Lee JH. A comparative cross-sectional study on cosmetic outcomes after single port or conventional laparoscopic surgery. Eur J Obstet Gynecol Reprod Biol 2013;167:104-9.

14. Tuschy B, Berlit S, Brade J, Sütterlin M, Hornemann A. Gynaecological laparoscopic surgery for benign conditions: do women care about incisions? Eur J Obstet Gynecol Reprod Biol 2013;169:84-7.

15. Kadar N, Reich H, Liu CY, Manko GF, Gimpelson R. Incisional hernias after major laparoscopic gynecologic procedures. Am J Obstet Gynecol 1993;168:1493-5.

16. Gunderson CC, Knight J, Ybanez-Morano J, Ritter C, Escobar $\mathrm{PF}$, Ibeanu $\mathrm{O}$, et al. The risk of umbilical hernia and other complications with laparoendoscopic single-site surgery. J Minim Invasive Gynecol 2012;19:40-5. 
17. Mayol J, Garcia-Aguilar J, Ortiz-Oshiro E, De-Diego Carmona JA, Fernandez-Represa JA. Risks of the minimal access approach for laparoscopic surgery: multivariate analysis of morbidity related to umbilical trocar insertion. World J Surg 1997;21: 529-33.

18. Bradford LS, Boruta DM. Laparoendoscopic single-site surgery in gynecology: a review of the literature, tools, and techniques. Obstet Gynecol Surv 2013;68:295-304.

19. Chang SK, Tay CW, Bicol RA, Lee YY, Madhavan K. A case-control study of single-incision versus standard laparoscopic cholecystectomy. World J Surg 2011;35:289-93.

20. Kim SM, Park EK, Jeung IC, Kim CJ, Lee YS. Abdominal, multi-port and single-port total laparoscopic hysterectomy: eleven-year trends comparison of surgical outcomes complications of 936 cases. Arch Gynecol Obstet 2015;291:1313-9.

21. Sandberg EM, la Chapelle CF, van den Tweel MM, Schoones JW, Jansen FW. Laparoendoscopic single-site surgery versus conventional laparoscopy for hysterectomy: a systematic review and meta-analysis. Arch Gynecol Obstet 2017;295:1089103.

22. Paek J, Kim SW, Lee SH, Lee M, Yim GW, Nam EJ, et al. Learning curve and surgical outcome for single-port access total laparoscopic hysterectomy in 100 consecutive cases. Gynecol Obstet Invest 2011;72:227-33.

23. Kho RM, Abrão MS. In search for the best minimally invasive hysterectomy approach for the large uterus: a review. Clin Obstet Gynecol 2017;60:286-95.

24. Smith K, Caceres A. Vaginal cuff closure in minimally invasive hysterectomy: a review of training, techniques, and materials. Cureus 2017;9:e1766.

25. Lee SW, Park EK, Lee SJ, Lee KH. Comparison study of consecutive 100 cases of single port vs. multiport laparoscopic myomectomy; technical point of view. J Obstet Gynaecol 2017;37:616-21.

26. Fader AN, Escobar PF. Laparoendoscopic single-site surgery (LESS) in gynecologic oncology: technique and initial report. Gynecol Oncol 2009;114:157-61.

27. Fagotti A, Boruta DM 2nd, Scambia G, Fanfani F, Paglia A,
Escobar PF. First 100 early endometrial cancer cases treated with laparoendoscopic single-site surgery: a multicentric retrospective study. Am J Obstet Gynecol 2012;206:353.e1-6.

28. Garrett LA, Boruta DM 2nd. Laparoendoscopic single-site radical hysterectomy: the first report of LESS type III hysterectomy involves a woman with cervical cancer. Am J Obstet Gynecol 2012;207:518.e1-2.

29. Boruta DM, Fagotti A, Bradford LS, Escobar PF, Scambia G, Kushnir CL, et al. Laparoendoscopic single-site radical hysterectomy with pelvic lymphadenectomy: initial multi-institutional experience for treatment of invasive cervical cancer. J Minim Invasive Gynecol 2014;21:394-8.

30. Escobar PF, Fader AN, Paraiso MF, Kaouk JH, Falcone T. Robotic-assisted laparoendoscopic single-site surgery in gynecology: initial report and technique. J Minim Invasive Gynecol 2009;16:589-91.

31. Iavazzo C, Minis EE, Gkegkes ID. Single-site port robotic-assisted hysterectomy: an update. J Robot Surg 2018;12:20113.

32. Lopez S, Mulla ZD, Hernandez L, Garza DM, Payne TN, Farnam RW. A comparison of outcomes between robotic-assisted, single-site laparoscopy versus laparoendoscopic single site for benign hysterectomy. J Minim Invasive Gynecol 2016; 23:84-8.

33. Jallad K, Walters MD. Natural orifice transluminal endoscopic surgery (NOTES) in gynecology. Clin Obstet Gynecol 2017;60:324-9.

34. Yang YS, Hur MH, Oh KY, Kim SY. Transvaginal natural orifice transluminal endoscopic surgery for adnexal masses. J Obstet Gynaecol Res 2013;39:1604-9.

35. Baekelandt J, De Mulder PA, Le Roy I, Mathieu C, Laenen A, Enzlin P, et al. Postoperative outcomes and quality of life following hysterectomy by natural orifice transluminal endoscopic surgery (NOTES) compared to laparoscopy in women with a non-prolapsed uterus and benign gynaecological disease: a systematic review and meta-analysis. Eur J Obstet Gynecol Reprod Biol 2017;208:6-15. 